EUROPEAN JOURNAL OF PURE AND APPLIED MATHEMATICS

Vol. 12, No. 2, 2019, 519-532

ISSN 1307-5543 - www.ejpam.com

Published by New York Business Global

\title{
General solution of linear partial differential equations modeling homogeneous diffusion-convection-reaction problems with Cauchy initial condition
}

\author{
Minoungou Youssouf ${ }^{1}$, Bagayogo Moussa ${ }^{1}$, Youssouf Paré ${ }^{1, *}$ \\ ${ }^{1}$ Département de Mathématiques, UFR/Sciences Exactes et Appliquées, Université Ouaga \\ I Pr Joseph Ki-Zerbo, Ouagadougou, Burkina-Faso
}

\begin{abstract}
In this paper, we propose the general solution of diffusion-convection-reaction homogeneous problems with condition initial of Cauchy, using the SBA numerical method. This method is based on the combination of the Adomian Decompositional Method(ADM), the successive approximations method and the Picard principle.
\end{abstract}

2010 Mathematics Subject Classifications: 47H14, 34G20, 47J25, 65J15

Key Words and Phrases: SBA method, Adomian Decompositional Method(ADM), homogeneous Diffusion-convection-reaction problem.

\section{Introduction}

Most physical, medical, biological,...,phenomena are modeled by integral equations, integro-differential equations, ordinary differential equations or by partial differential equations. Generally it is very difficult, or impossible to determine their analytical solutions. In this paper, we propose a general solution of linear homogeneous diffusion, convection and reaction equations with Cauchy initial condition, using the SBA numerical method.

\section{The numerical SBA method}

Let us consider the following fonctional equation:

$$
A u=f
$$

Where $A: H \rightarrow H$, is an operator not necessarly linear and $H$ is a Hilbert space adequatly chosen given the operator $A, f$ is given function and $u$ the unnown function.

${ }^{*}$ Corresponding author.

DOI: https://doi.org/10.29020/nybg.ejpam.v12i2.3411

Email addresses: m.youl@yahoo.fr (Y. Minoungou), moussabagayogo94@gmail.com (M. Bagayogo), pareyoussouf@yahoo.fr (Y. Paré) 
Let :

$$
A=L+R+N
$$

Where $L$ is an invertible operator in the Adomian "sense", $R$ the linear remainder and $N$ a nonlinear operator. The equation (2) therefore becomes:

$$
L u+R u+N u=f
$$

$\Leftrightarrow$

$$
u=\theta+L^{-1}(f)-L^{-1}(R u)-L^{-1}(N u)
$$

Where $\theta$ is such that $L(\theta)=0$. The equation (3) is the Adomian canonical forme, using the successsive approximations [3] we get :

$$
u^{k}=\theta+L^{-1}(f)-L^{-1}\left(R u^{k}\right)-L^{-1}\left(N u^{k-1}\right) ; k \geq 1
$$

This yields the following Adomian algorithm [5]

$$
\left\{\begin{array}{l}
u_{0}^{k}=\theta+L^{-1}(f)-L^{-1}\left(N u^{k-1}\right) ; k \geq 1 \\
u_{n+1}^{k}=-L^{-1}\left(R u_{n}^{k}\right) ; n \geq 0
\end{array}\right.
$$

The Picard principle is then applied to the equation (5) let $u^{0}$ be such that $N\left(u^{0}\right)=0$, for $k=1$, we get :

$$
\left\{\begin{array}{l}
u_{0}^{1}=\theta+L^{-1}(f)+L^{-1}\left(N u^{0}\right) \\
u_{n+1}^{1}=-L^{-1}\left(R u_{n}^{1}\right) ; n \geq 0
\end{array}\right.
$$

If the series $\left(\sum_{n=0}^{+\infty} u_{n}^{1}\right)$ converges, then $u^{1}=\sum_{n=0}^{+\infty} u_{n}^{1}$. For $k=2$, we get:

$$
\left\{\begin{array}{l}
u_{0}^{2}=\theta+L^{-1}(f)+L^{-1}\left(N u^{1}\right) \\
u_{n+1}^{2}=L^{-1}\left(R u_{n}^{2}\right) ; n \geq 0
\end{array}\right.
$$

If the series $\left(\sum_{n=0}^{+\infty} u_{n}^{2}\right)$ converges, then $u^{2}=\sum_{n=0}^{+\infty} u_{n}^{2}$. This process is repeated to $k$.

If the series $\left(\sum_{n=0}^{+\infty} u_{n}^{k}\right)$ converges, then $u^{2}=\sum_{n=0}^{+\infty} u_{n}^{k}$, therefore $u=\lim _{k \rightarrow+\infty} u^{k}$ is the solution of the equation (2)

At each stape $k \geq 1$, we make sure that : $N\left(u^{k}\right)=0$.

\subsection{A diffusion model}

Let us consider the following diffusion model Cauchy initial condition $[1,6]$

$$
\left\{\begin{array}{l}
\frac{\partial u(t, x)}{\partial t}=\varepsilon \frac{\partial^{2} u(t, x)}{\partial x^{2}}, 0<\varepsilon \ll 1 \\
u(0, x)=\sin \omega x, \omega>0
\end{array}\right.
$$


where $(t, x) \in \Omega=\left[0,+\infty\left[\times \mathbb{R}\right.\right.$ and $u \in C^{2}(\Omega)$.

Appliying the SBA method to (8) at the step $k \geq 0$, we obtain the following algorithm $[2,4]$

$$
\left(P_{S B A}\right):\left\{\begin{array}{l}
u_{0}^{k}(t, x)=\sin \omega x \\
u_{n+1}^{k}(t, x)=\varepsilon \int_{0}^{t} \frac{\partial^{2} u_{n}^{k}(s, x)}{\partial x^{2}} d s, n \geq 0
\end{array}\right.
$$

Let us calculate the following terms: $u_{1}^{k}(t, x), u_{2}^{k}(t, x), u_{3}^{k}(t, x), \ldots$

$$
\left\{\begin{aligned}
u_{0}^{k}(t, x) & =\sin \omega x \\
u_{1}^{k}(t, x) & =-\varepsilon \omega^{2} t \sin \omega x \\
u_{2}^{k}(t, x) & =\frac{\left(\varepsilon \omega^{2} t\right)^{2}}{2 !} \sin \omega x \\
u_{3}^{k}(t, x) & =\frac{\left(-\varepsilon \omega^{2} t\right)^{3}}{3 !} \sin \omega x \\
\ldots & \ldots \\
u_{n}^{k}(t, x) & =\frac{\left(-\varepsilon \omega^{2} t\right)^{n}}{n !} \sin \omega x
\end{aligned}\right.
$$

Then we obtain :

$$
u^{k}(t, x)=\sin \omega x \sum_{n=0}^{+\infty} \frac{\left(-\varepsilon \omega^{2} t\right)^{n}}{n !}=\exp \left(-\varepsilon \omega^{2} t\right) \sin \omega x
$$

And the exact solution of (8) is ;

$$
u(t, x)=\exp \left(-\varepsilon \omega^{2} t\right) \sin \omega x
$$

Proposition 1. The exact solution of the following diffusion problem with Cauchy initial condition :

$$
\left\{\begin{array}{l}
\frac{\partial u(t, x)}{\partial t}=\varepsilon \frac{\partial^{2} u(t, x)}{\partial x^{2}}, \varepsilon>0 \\
u(0, x)=\varphi(\alpha x), \alpha \neq 0
\end{array}\right.
$$

is

$$
u(t, x)=\exp \left(-\varepsilon \alpha^{2} t\right) \varphi(\alpha x)
$$

where $(t, x) \in \Omega=\left[0,+\infty\left[\times \mathbb{R}, u \in C^{2}(\Omega), \varphi \in C^{2}(\mathbb{R})\right.\right.$ and $\varphi$ verifie the relation :

$$
\varphi(x)=A \cos x+B \sin x, A, B \in \mathbb{R} .
$$

Proof. Let us consider $u(t, x)=\exp \left(-\varepsilon \alpha^{2} t\right) \varphi(\alpha x)$. where $(t, x) \in \Omega=\left[0,+\infty\left[\times \mathbb{R}, u \in C^{2}(\Omega), \varphi \in C^{2}(\mathbb{R})\right.\right.$

We obtain :

$$
\begin{aligned}
\frac{\partial}{\partial t} \exp \left(-\varepsilon \alpha^{2} t\right) \varphi(\alpha x)-\varepsilon \frac{\partial^{2}}{\partial x^{2}} \exp \left(-\varepsilon \alpha^{2} t\right) \varphi(\alpha x) & =\left(-\varepsilon \alpha^{2}\right)\left(\varphi(\alpha x)+\varphi^{\prime \prime}(\alpha x)\right) \exp \left(-\varepsilon \alpha^{2} t\right) \\
& =0
\end{aligned}
$$




$$
\begin{aligned}
& \Leftrightarrow \varphi(\alpha x)+\varphi^{\prime \prime}(\alpha x)=0 \\
& \Leftrightarrow \varphi(\alpha x)=A \cos (\alpha x)+B \sin (\alpha x)
\end{aligned}
$$

Hence $u(t, x)=\exp \left(-\varepsilon \alpha^{2} t\right)(A \cos (\alpha x)+B \sin (\alpha x))$ and $u(0, x)=\varphi(\alpha x)$

Then $u(t, x)=\exp \left(-\varepsilon \alpha^{2} t\right)(A \cos (\alpha x)+B \sin (\alpha x))$ is the general solution of (10) with $\varphi(\alpha x)=A \cos (\alpha x)+B \sin (\alpha x), A \in \mathbb{R}, B \in \mathbb{R}$.

\subsection{A convection model}

Let us consider the following convection model with Cauchy initial [6-8]

$$
\left\{\begin{array}{l}
\frac{\partial u(t, x)}{\partial t}=\lambda \frac{\partial u(t, x)}{\partial x} ; \lambda>0 \\
u(0, x)=\cos \alpha x ; \alpha \neq 0
\end{array}\right.
$$

where $(t, x) \in \Omega=\left[0,+\infty\left[\times \mathbb{R}, u \in C^{1}(\Omega)\right.\right.$ et $\varphi \in C^{1}(\mathbb{R})$.

Appliying the SBA method to (13) at the step $k \geq 0$.

We obtain the following algorithm :

$$
\left(P_{S B A}\right):\left\{\begin{array}{l}
u_{0}^{k}(t, x)=\cos \alpha x \\
u_{n+1}^{k}(t, x)=\lambda \int_{0}^{t} \frac{\partial u_{n}^{k}(s, x)}{\partial x} d s, n \geq 0
\end{array}\right.
$$

Let us calculate the following terms: $u_{1}^{k}(t, x), u_{2}^{k}(t, x), u_{3}^{k}(t, x), \ldots$

$$
\left\{\begin{array}{l}
u_{0}^{k}(t, x)=\cos \alpha x \\
u_{1}^{k}(t, x)=-t \alpha \lambda \sin \alpha x \\
u_{2}^{k}(t, x)=-\frac{(t \alpha \lambda)^{2}}{2 !} \cos \alpha x \\
u_{3}^{k}(t, x)=\frac{(\alpha \lambda t)^{3}}{3 !} \sin \alpha x \\
u_{4}^{k}(t, x)=\frac{(\alpha \lambda t)^{4}}{4 !} \cos \alpha x \\
u_{5}^{k}(t, x)=-\frac{(\alpha \lambda t)^{5}}{5 !} \sin \alpha x \\
u_{6}^{k}(t, x)=-\frac{(\alpha \lambda t)^{6}}{6 !} \cos \alpha x \\
\cdots \\
u_{2 n}^{k}(t, x)=(-1)^{n} \frac{(\alpha \lambda t)^{2 n}}{(2 n) !} \cos \alpha x ; n \geq 0 \\
u_{2 n+1}^{k}(t, x)=-(-1)^{n} \frac{(\alpha \lambda t)^{2 n+1}}{(2 n+1) !} \sin \alpha x ; n \geq 0
\end{array}\right.
$$

Then 


$$
u^{k}(t, x)=\lim _{n \rightarrow+\infty} \cos \alpha x \sum_{n=0}^{m}(-1)^{n} \frac{(\alpha \lambda t)^{2 n}}{(2 n) !}-\lim _{n \rightarrow+\infty} \sin \alpha x \sum_{n=0}^{m}(-1)^{n} \frac{(\alpha \lambda t)^{2 n+1}}{(2 n+1) !}
$$

We obtain the exact solution of the problem (13)

$$
u(t, x)=\cos (\alpha(x+\lambda t)) .
$$

Proposition 2. The exact solution of the following convection model with Cauchy initial condition

$$
\left\{\begin{array}{l}
\frac{\partial u(t, x)}{\partial t}=\lambda \frac{\partial u(t, x)}{\partial x}, \varepsilon>0, \lambda>0 \\
u(0, x)=\varphi(\alpha x)
\end{array}\right.
$$

is

$$
u(t, x)=\varphi(\alpha(x+\lambda t))
$$

where $(t, x) \in \Omega=\left[0,+\infty\left[\times \mathbb{R}, u \in C^{1}(\Omega), \varphi \in C^{1}(\mathbb{R})\right.\right.$.

Proof. Let us consider $u(t, x)=\varphi(\alpha(x+\lambda t))$.

We obtain :

$$
\begin{aligned}
\frac{\partial}{\partial t} \varphi(\alpha(x+\lambda t))-\lambda \frac{\partial}{\partial x} \varphi(\alpha(x+\lambda t)) & =\alpha \lambda \varphi^{\prime}(\alpha(x+\lambda t))-\alpha \lambda \varphi^{\prime}(\alpha(x+\lambda t)) \\
& =0
\end{aligned}
$$

$\forall \varphi \in C^{2}(\mathbb{R})$ and $u(0, x)=\varphi(\alpha x)$

In this case, it is necessary and sufficient that the function $\varphi \in C^{1}(\mathbb{R})$.

Hence $u(t, x)=\varphi(\alpha(x+\lambda t))$ is the general solution of (16).

\subsection{A reaction model}

Let us consider the following reaction model Cauchy type [6]

$$
\left\{\begin{array}{l}
\frac{\partial u(t, x)}{\partial t}=\gamma u(t, x), \quad \gamma>0 \\
u(0, x)=\sin \alpha x
\end{array}\right.
$$

where $(t, x) \in \Omega=\left[0,+\infty\left[\times \mathbb{R}, u \in C^{1}(\Omega)\right.\right.$ et $\varphi \in C^{1}(\mathbb{R})$.

Appliying the SBA algorithm to (18) at the step $k \geq 0$, we obtain the following algorithm :

$$
\left(P_{S B A}\right):\left\{\begin{array}{l}
u_{0}^{k}(t, x)=\sin \alpha x \\
u_{n+1}^{k}(t, x)=\gamma \int_{0}^{t} u_{n}^{k}(s, x) d s, n \geq 0
\end{array}\right.
$$


Let us calculate the following terms: $u_{1}^{k}(t, x), u_{2}^{k}(t, x), u_{3}^{k}(t, x), \ldots$

$$
\left\{\begin{array}{l}
u_{0}^{k}(t, x)=\sin \alpha x \\
u_{1}^{k}(t, x)=\gamma t \sin \alpha x \\
u_{2}^{k}(t, x)=\frac{(\gamma t)^{2}}{2 !} \sin \alpha x \\
u_{3}^{k}(t, x)=\frac{(\gamma t)^{3}}{3 !} \sin \alpha x \\
u_{4}^{k}(t, x)=\frac{(\gamma t)^{4}}{4 !} \sin \alpha x \\
u_{5}^{k}(t, x)=\frac{(\gamma t)^{5}}{5 !} \sin \alpha x \\
\ldots \\
u_{n}^{k}(t, x)=\frac{(\gamma t)^{n}}{n !} \sin \alpha x
\end{array}\right.
$$

$u^{k}(t, x)=u_{0}^{k}(t, x)+u_{1}^{k}(t, x)+u_{2}^{k}(t, x)+\ldots$

$u^{k}(t, x)=\sin \alpha x \sum_{n=0}^{+\infty} \frac{(\gamma t)^{n}}{n !}$

Then

$$
u^{k}(t, x)=\exp (\gamma t) \sin \alpha x
$$

we obtain the exact solution of the problem (18)

$$
u(t, x)=\exp (\gamma t) \sin \alpha x
$$

Proposition 3. The exact solution of the following reaction problem Cauchy type:

$$
\left\{\begin{array}{l}
\frac{\partial u(t, x)}{\partial t}=\gamma u(t, x), \quad \gamma>0 \\
u(0, x)=\varphi(\alpha x), \quad \alpha \neq 0
\end{array}\right.
$$

is

$$
u(t, x)=\exp (\gamma t) \varphi(\alpha x)
$$

where $(t, x) \in \Omega=\left[0,+\infty\left[\times \mathbb{R}, u \in C^{1}(\Omega), \varphi \in C^{1}(\mathbb{R})\right.\right.$.

Proof. Let us consider $u(t, x)=\exp (\gamma t) \varphi(\alpha x)$, we obtain :

$$
\begin{aligned}
\frac{\partial}{\partial t} \exp (\gamma t) \varphi(\alpha x)-\gamma \exp (\gamma t) \varphi(\alpha x) & =\exp (\gamma t)(\gamma-\gamma) \varphi(\alpha x) \\
& =0
\end{aligned}
$$

and $u(0, x)=\varphi(\alpha x) \Longleftrightarrow \forall \varphi \in C^{1}(\mathbb{R})$.

In this case,it is necessary and sufficient that the function $\varphi \in C^{1}(I)$ and $I \subset \mathbb{R}$ or $I=\mathbb{R}$, hence the general solution of $(21)$ is $u(t, x)=\exp (\gamma t) \varphi(\alpha x)$. 


\subsection{A diffusion-convection model}

Let us consider the following type of Cauchy linear equation :

$$
(D): \begin{cases}\frac{\partial u(t, x)}{\partial t} & =\varepsilon \frac{\partial^{2} u(t, x)}{\partial x^{2}}+\lambda \frac{\partial u(t, x)}{\partial x} ; 0<\varepsilon \ll 1, \lambda>0, t>0, x \in \mathbb{R} \\ u(0, x) & =\sin x\end{cases}
$$

Applying the algorithm SBA to $(D)$, we have :

$$
\left(P_{S B A}\right):\left\{\begin{array}{l}
u_{0}^{k}(t, x)=\sin x \\
u_{n+1}^{k}(t, x)=\int_{0}^{t}\left(\varepsilon \frac{\partial^{2} u_{n}^{k}(s, x)}{\partial x^{2}}+\lambda \frac{\partial u_{n}^{k}(s, x)}{\partial x}\right) d s ; n \geq 0
\end{array}\right.
$$

Let us determinate the following terms: $u_{0}^{k}(t, x), u_{1}^{k}(t, x), u_{2}^{k}(t, x), u_{3}^{k}(t, x), \ldots, u_{n}^{k}(t, x)$.

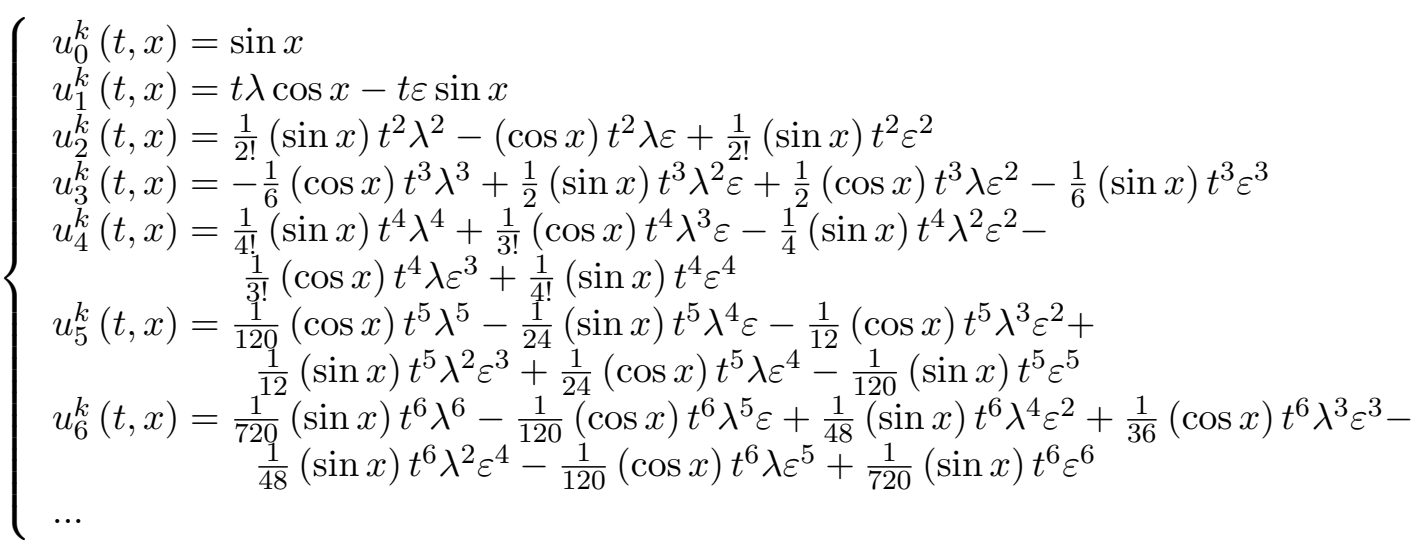

Step by step, we then deduct : 


$$
\left\{\begin{aligned}
u^{k}(t, x) \simeq \quad & \sin x\left(1-\varepsilon t+\frac{(\varepsilon t)^{2}}{2 !}--\frac{(\varepsilon t)^{3}}{3 !}+\ldots\right) \\
& -\frac{(\lambda t)^{2}}{2 !} \sin x\left(1-\varepsilon t+\frac{(\varepsilon t)^{2}}{2 !}--\frac{(\varepsilon t)^{3}}{3 !}+\ldots\right) \\
& +\frac{(\lambda t)^{4}}{4 !} \sin x\left(1-\varepsilon t+\frac{(\varepsilon t)^{2}}{2 !}-\frac{(\varepsilon t)^{3}}{3 !}+\ldots\right) \\
& -\frac{(\lambda t)^{6}}{6 !} \sin x\left(1-\varepsilon t+\frac{(\varepsilon t)^{2}}{2 !}--\frac{(\varepsilon t)^{3}}{3 !}+\ldots\right) \\
& \ldots \\
& +\lambda t \cos x\left(1-\varepsilon t+\frac{(\varepsilon t)^{2}}{2 !}-\frac{(\varepsilon t)^{3}}{3 !}+\ldots\right) \\
& -\frac{(\lambda t)^{3}}{3 !} \cos x\left(1-\varepsilon t+\frac{(\varepsilon t)^{2}}{2 !}--\frac{(\varepsilon t)^{3}}{3 !}+\ldots\right) \\
& +\frac{(\lambda t)^{5}}{5 !} \cos x\left(1-\varepsilon t+\frac{(\varepsilon t)^{2}}{2 !}--\frac{(\varepsilon t)^{3}}{3 !}+\ldots\right) \\
& \ldots
\end{aligned}\right.
$$

Then, we obtain

$$
\left\{\begin{aligned}
u^{k}(t, x) \simeq \quad & \sin x\left(1-\varepsilon t+\frac{(\varepsilon t)^{2}}{2 !}-\frac{(\varepsilon t)^{3}}{3 !}+\ldots\right)\left(1-\frac{(\lambda t)^{2}}{2 !}+\frac{(\lambda t)^{4}}{4 !}+\ldots\right)+ \\
& \cos x\left(1-\varepsilon t+\frac{(\varepsilon t)^{2}}{2 !}--\frac{(\varepsilon t)^{3}}{3 !}+\ldots\right)\left(\lambda t-\frac{(\lambda t)^{3}}{3 !}+\frac{(\lambda t)^{5}}{5 !}-\ldots\right)
\end{aligned}\right.
$$

In a recurcive way, we obtain :

$$
u^{k}(t, x)=\lim _{n \rightarrow+\infty} \sin x \sum_{p=0}^{n} \frac{(-\varepsilon t)^{p}}{p !} \sum_{p=0}^{n}(-1)^{p} \frac{(\lambda t)^{2 p}}{(2 p) !}+\cos x \sum_{p=0}^{n} \frac{(-\varepsilon t)^{p}}{p !} \sum_{p=0}^{n}(-1)^{p} \frac{(\lambda t)^{2 p+1}}{(2 p+1) !}
$$

Therefore, we get

$$
u^{k}(t, x)=\exp (-\varepsilon t)(\sin x \cos \lambda t+\sin \lambda t \cos x) .
$$

$\Rightarrow$

$$
u^{k}(t, x)=\exp (-\varepsilon t) \sin (x+\lambda t)
$$

So, the exact solution exact of $(D)$ is

$$
u(t, x)=\exp (-\varepsilon t) \sin (x+\lambda t) .
$$


Proposition 4. The exact solution of the following reaction problem Cauchy type:

$$
\left(P_{4}\right): \begin{cases}\frac{\partial u(t, x)}{\partial t} & =\varepsilon \frac{\partial^{2} u(t, x)}{\partial x^{2}}+\lambda \frac{\partial u(t, x)}{\partial x} ; 0<\varepsilon \ll 1, \lambda>0, t>0, x \in \mathbb{R} \\ u(0, x) & =\varphi(x)\end{cases}
$$

is

$$
u(t, x)=\exp (-\varepsilon t) \varphi(x+\lambda t)
$$

where $(t, x) \in \Omega=\left[0,+\infty\left[\times \mathbb{R}, u \in C^{2}(\Omega), \varphi \in C^{2}(\mathbb{R})\right.\right.$.

Proof. Let us consider $u(t, x)=\exp (-\varepsilon t) \varphi(x+\lambda t)$ We obtain :

$$
\begin{aligned}
\frac{\partial}{\partial t} \exp & (-\varepsilon t) \varphi(x+\lambda t)-\varepsilon \frac{\partial^{2}}{\partial x^{2}} \exp (-\varepsilon t) \varphi(x+\lambda t)-\lambda \frac{\partial}{\partial x} \exp (-\varepsilon t) \varphi(x+\lambda t) \\
& =\exp (-\varepsilon t)\left(-\varepsilon \varphi(x+\lambda t)+\lambda \varphi^{\prime}(x+\lambda t)-\varepsilon \varphi^{\prime \prime}(x+\lambda t)-\lambda \varphi^{\prime}(x+\lambda t)\right) \\
& =0 \\
& \Rightarrow \varphi^{\prime \prime}(x+\lambda t)+\varphi(x+\lambda t)=0 \\
& \Rightarrow \varphi(x+\lambda t)=A \cos (x+\lambda t)+B \sin (x+\lambda t)
\end{aligned}
$$

where $A, B \in \mathbb{R}$ and $u(0, x)=\varphi(x) \Longleftrightarrow \forall \varphi \in C^{1}(\mathbb{R})$

In this case, it is necessary and sufficient that the function $\varphi \in C^{1}(I)$ where $I \subset \mathbb{R}$ or $I=\mathbb{R}$, hence the general solution of $\left(P_{4}\right)$ is $u(t, x)=\exp (\gamma t) \varphi(\alpha x)$.

\subsection{A reaction model}

Proposition 5. The exact solution of the following reaction problem Cauchy type:

$$
(E)\left\{\begin{array}{l}
\frac{\partial u(t, x)}{\partial t}=\gamma u(t, x), \quad \gamma>0 \\
u(0, x)=\varphi(\alpha x), \quad \alpha \neq 0
\end{array}\right.
$$

is

$$
u(t, x)=\exp (\gamma t) \varphi(\alpha x)
$$

where $(t, x) \in \Omega=\left[0,+\infty\left[\times \mathbb{R}, u \in C^{1}(\Omega), \varphi \in C^{1}(\mathbb{R})\right.\right.$.

Proof. Let us consider $u(t, x)=\exp (\gamma t) \varphi(\alpha x)$.

We obtain :

$$
\begin{aligned}
\frac{\partial}{\partial t} \exp (\gamma t) \varphi(\alpha x)-\gamma \exp (\gamma t) \varphi(\alpha x) & =\exp (\gamma t)(\gamma-\gamma) \varphi(\alpha x) \\
& =0
\end{aligned}
$$

and $u(0, x)=\varphi(\alpha x)$

$\Longleftrightarrow \forall \varphi \in C^{1}(I)$ where $I \subset \mathbb{R}$ or $I=\mathbb{R}$

In this case, it is necessary and sufficient that the function $\varphi \in C^{1}(I)$ where $I \subset \mathbb{R}$ or $I \subset \mathbb{R}$, hence the general solution of $(E)$ is $u(t, x)=\exp (\gamma t) \varphi(\alpha x)$. 


\subsection{A diffusion-reaction model}

Let us consider the following diffision-reaction problem Cauchy type:

$$
(F)\left\{\begin{array}{l}
\frac{\partial u(t, x)}{\partial t}=\varepsilon \frac{\partial^{2} u(t, x)}{\partial x^{2}}+\gamma u(t, x), \quad 0<\varepsilon \ll 1, \gamma>0 \\
u(0, x)=\sin \alpha x, \quad \alpha \neq 0
\end{array}\right.
$$

where $(t, x) \in \Omega=\left[0,+\infty\left[\times \mathbb{R}, u \in C^{2}(\Omega)\right.\right.$ et $\varphi \in C^{2}(\mathbb{R})$.

Appliying the SBA method at the step $k \geq 0$, we obtain the following algorithm :

$$
\left(P_{S B A}\right):\left\{\begin{array}{l}
u_{0}^{k}(t, x)=\sin \alpha x \\
u_{n+1}^{k}(t, x)=\varepsilon \int_{0}^{t} \frac{\partial^{2} u(s, x)}{\partial x^{2}} d s+\gamma \int_{0}^{t} u_{n}^{k}(s, x) d s ; n \geq 0
\end{array}\right.
$$

Let us calculate some terms:

We obtain at the same way:

$$
u^{k}(t, x)=\exp \left(\left(\gamma-\varepsilon \alpha^{2}\right) t\right)(\sin \alpha x \cos \alpha \lambda t+\cos \alpha x \sin \alpha \lambda t)
$$

we obtain the exact solution of the problem $(F)$ :

$$
u(t, x)=\exp \left(\left(\gamma-\varepsilon \alpha^{2}\right) t\right) \sin \alpha(x+\lambda t)
$$

Proposition 6. The exact solution of the following diffision-reaction problem Cauchy type

$$
\left(P_{6}\right)\left\{\begin{array}{l}
\frac{\partial u(t, x)}{\partial t}=\varepsilon \frac{\partial^{2} u(t, x)}{\partial x^{2}}+\gamma u(t, x) ; \varepsilon>0, \lambda>0 \\
u(0, x)=\varphi(\alpha x)
\end{array}\right.
$$

$i s$

$$
u(t, x)=\exp \left(\left(\gamma-\varepsilon \alpha^{2}\right) t\right) \varphi(\alpha(x+\lambda t))
$$

where $(t, x) \in \Omega=\left[0,+\infty\left[\times \mathbb{R}, u \in C^{2}(\Omega), \varphi \in C^{2}(\mathbb{R})\right.\right.$ and $\varphi$ verifie the relation :

$$
\varphi(x)=A \cos x+B \sin x ; A, B \in \mathbb{R}
$$

Proof. Let us consider $u(t, x)=\exp \left(-\varepsilon \alpha^{2} t\right) \varphi(\alpha(x+\lambda t))$.

We obtain :

$$
\begin{array}{r}
\frac{\partial}{\partial t} \exp \left(-\varepsilon \alpha^{2} t\right) \varphi(\alpha(x+\lambda t))-\varepsilon \frac{\partial^{2}}{\partial x^{2}} \exp \left(-\varepsilon \alpha^{2} t\right) \varphi(\alpha(x+\lambda t)) \\
-\lambda \frac{\partial}{\partial x} \exp \left(-\varepsilon \alpha^{2} t\right) \varphi(\alpha(x+\lambda t)) \\
=\begin{array}{r}
\exp \left(-\varepsilon \alpha^{2} t\right)\left(-\varepsilon \alpha^{2} \varphi(\alpha(x+\lambda t))+\alpha \lambda \varphi^{\prime}(\alpha(x+\lambda t))\right. \\
\left.-\varepsilon \alpha^{2} \varphi^{\prime \prime}(\alpha(x+\lambda t))-\lambda \alpha \varphi^{\prime}(\alpha(x+\lambda t))\right)
\end{array}
\end{array}
$$




$$
=0
$$

$\Leftrightarrow \varphi(\alpha(x+\lambda t))+\varphi^{\prime \prime}(\alpha(x+\lambda t))=0$

$\Rightarrow \varphi(\alpha(x+\lambda t))=A \cos (\alpha(x+\lambda t))+B \sin (\alpha(x+\lambda t)), A, B \in \mathbb{R}$

Hence the general solution of $\left(P_{6}\right)$ is of the form :

$u(t, x)=\exp \left(-\varepsilon \alpha^{2} t\right) \varphi(\alpha(x+\lambda t))$,

with $\varphi(\alpha(x+\lambda t))=A \cos (\alpha(x+\lambda t))+B \sin (\alpha(x+\lambda t))$ and $u(0, x)=\varphi(\alpha x)$.

\subsection{A diffusion-convection-reaction problem Cauchy type}

Let us consider the following diffision-convection-reaction problem Cauchy type:

$$
(H)\left\{\begin{array}{l}
\frac{\partial u(t, x)}{\partial t}=\varepsilon \frac{\partial^{2} u(t, x)}{\partial^{2} x}+\lambda \frac{\partial u(t, x)}{\partial x}+\gamma u(t, x), \varepsilon>0, \lambda>0, \gamma>0 \\
u(0, x)=\sin \alpha x
\end{array}\right.
$$

where $(t, x) \in \Omega=\left[0,+\infty\left[\times \mathbb{R}, u \in C^{2}(\Omega)\right.\right.$ et $\varphi \in C^{2}(\mathbb{R})$.

Appliying the SBA method at the step $k \geq 0$, we obtain the following algorithm :

$$
\left(P_{S B A}\right):\left\{\begin{array}{l}
u_{0}^{k}(t, x)=\sin \alpha x \\
u_{n+1}^{k}(t, x)=\varepsilon \int_{0}^{t} \frac{\partial^{2} u(s, x)}{\partial^{2} x} d s+\lambda \frac{\partial u(t, x)}{\partial x}+\gamma \int_{0}^{t} u_{n}^{k}(s, x) d s, n \geq 0
\end{array}\right.
$$

Let us calculate the following terms: $u_{1}^{k}(t, x), u_{2}^{k}(t, x), u_{3}^{k}(t, x), \ldots$

Let us consider the following Cauchy linear equation :

$$
\left(P_{3}\right):\left\{\begin{array}{l}
\frac{\partial u(t, x)}{\partial t}=\varepsilon \frac{\partial u(t, x)}{\partial x}+\mu u(t, x) ; 0<\varepsilon \ll 1, \mu>0, t>0, x \in \mathbb{R} \\
u(0, x)=\cos x
\end{array}\right.
$$

Applying the algorithm $S B A$ to $\left(P_{3}\right)$, we have :

$$
P_{S B A}:\left\{\begin{array}{l}
u_{0}^{k}(t, x)=\cos x \\
u_{n+1}^{k}(t, x)=\int_{0}^{t}\left(\varepsilon \frac{\partial u_{n}^{k}(s, x)}{\partial x}+\mu u_{n}^{k}(s, x)\right) d s ; n \geq 0
\end{array}\right.
$$

Let us calculate the following terms : 

$u_{0}^{k}(t, x), u_{1}^{k}(t, x), u_{2}^{k}(t, x), u_{3}^{k}(t, x), u_{4}^{k}(t, x), u_{5}^{k}(t, x), \ldots$

$$
\left\{\begin{aligned}
& u_{0}^{k}(t, x)= \cos x \\
& u_{1}^{k}(t, x)= t \mu \cos x-t \varepsilon \sin x \\
& u_{2}^{k}(t, x)= \frac{(t \mu)^{2}}{2} \cos x-\mu \varepsilon t^{2} \sin x-\frac{(t \varepsilon)^{2}}{2} \cos x \\
& u_{3}^{k}(t, x)= \frac{(t \mu)^{3}}{6} \cos x-\frac{1}{2} t^{3} \mu^{2} \varepsilon \sin x-\frac{1}{2} t^{3} \mu \varepsilon^{2} \cos x+\frac{(t \varepsilon)^{3}}{6} \sin x \\
& u_{4}^{k}(t, x)= \frac{(t \mu)^{4}}{24} \cos x-\frac{1}{6} t^{4} \mu^{3} \varepsilon \sin x-\frac{1}{4} t^{4} \mu^{2} \varepsilon^{2} \cos x+ \\
& \frac{1}{6} t^{4} \mu \varepsilon^{3} \sin x+\frac{(t \varepsilon)^{4}}{24} \cos x \\
& u_{5}^{k}(t, x)= \frac{(t \mu)^{5}}{120} \cos x-\frac{1}{24} t^{5} \mu^{4} \varepsilon \sin x-\frac{1}{12} t^{5} \mu^{3} \varepsilon^{2}(\cos x)+ \\
& \frac{1}{12} t^{5} \mu^{2} \varepsilon^{3} \sin x+\frac{1}{24} t^{5} \mu \varepsilon^{4} \cos x-\frac{(t \varepsilon)^{5}}{120} \sin x \\
& \ldots
\end{aligned}\right.
$$

Step by step, we then deduct

$$
\left\{\begin{array}{r}
u^{k}(t, x) \simeq\left(\begin{array}{l}
\left.1-\frac{(\varepsilon t)^{2}}{2}+\frac{(\varepsilon t)^{4}}{4 !}-\ldots\right)\left(1+\mu t+\frac{(\mu t)^{2}}{2 !}+\frac{(\mu t)^{3}}{3 !}+\ldots\right) \cos x- \\
\varepsilon t-\frac{(\varepsilon t)^{3}}{3 !}+\frac{(\varepsilon t)^{5}}{5 !}-. .
\end{array}\right)\left(1+\mu t+\frac{(\mu t)^{2}}{2 !}+\frac{(\mu t)^{3}}{3 !}+\ldots\right) \sin x
\end{array}\right.
$$

In a recurcive way, we otain:

$$
u^{k}(t, x)=\lim _{n \rightarrow+\infty} \sum_{p=0}^{n}(-1)^{p} \frac{(\varepsilon t)^{2 p}}{(2 p) !} \sum_{p=0}^{n} \frac{(\mu t)^{p}}{p !} \cos x+\sum_{p=0}^{n}(-1)^{p} \frac{(\varepsilon t)^{2 p+1}}{(2 p+1) !} \sum_{p=0}^{n} \frac{(\mu t)^{p}}{p !} \sin x
$$

then,we get :

$$
u^{k}(t, x)=\exp (\mu t) \cos (\varepsilon t+x)
$$

And the exact solution of $\left(P_{3}\right)$ is

$$
u(t, x)=\lim _{k \rightarrow+\infty} u^{k}(t, x)=\exp (\mu t) \cos (\varepsilon t+x)
$$

Proposition 7. The exact solution of the following diffision-convection problem Cauchy type

$$
\left(P_{7}\right)\left\{\begin{array}{l}
\frac{\partial u(t, x)}{\partial t}=\varepsilon \frac{\partial^{2} u(t, x)}{\partial^{2} x}+\lambda \frac{\partial u(t, x)}{\partial x}+\gamma u(t, x), \varepsilon>0, \lambda>0, \gamma>0 \\
u(0, x)=\varphi(\alpha x)
\end{array}\right.
$$


is

$$
u(t, x)=\exp \left(\left(\gamma-\varepsilon \alpha^{2}\right) t\right) \varphi(\alpha(x+\lambda t))
$$

where $(t, x) \in \Omega=\left[0,+\infty\left[\times \mathbb{R}, u \in C^{2}(\Omega), \varphi \in C^{2}(\mathbb{R})\right.\right.$ and $\varphi$ verifie the relation:

$$
\varphi(x)=A \cos x+B \sin x, A, B \in \mathbb{R}
$$

Proof. Let us consider $u(t, x)=\exp \left(\left(\gamma-\varepsilon \alpha^{2}\right) t\right) \varphi(\alpha(x+\lambda t))$

We get :

$$
\begin{aligned}
& \frac{\partial \exp \left(\left(\gamma-\varepsilon \alpha^{2}\right) t\right) \varphi(\alpha(x+\lambda t))}{\partial t}-\varepsilon \frac{\partial^{2} \exp \left(\left(\gamma-\varepsilon \alpha^{2}\right) t\right) \varphi(\alpha(x+\lambda t))}{\partial^{2} x}- \\
& \lambda \frac{\partial \exp \left(\left(\gamma-\varepsilon \alpha^{2}\right) t\right) \varphi(\alpha(x+\lambda t))}{\partial x}-\gamma \exp \left(\left(\gamma-\varepsilon \alpha^{2}\right) t\right) \varphi(\alpha(x+\lambda t)) \\
& =\exp \left(\left(\gamma-\varepsilon \alpha^{2}\right) t\right)\left[\left(\gamma-\varepsilon \alpha^{2}\right) \varphi(\alpha(x+\lambda t))+\alpha \lambda \varphi^{\prime}(\alpha(x+\lambda t))\right]+ \\
& \exp \left(\left(\gamma-\varepsilon \alpha^{2}\right) t\right)\left[\varepsilon \alpha^{2} \varphi^{\prime \prime}(\alpha(x+\lambda t))-\lambda \alpha \varphi^{\prime}(\alpha(x+\lambda t))-\gamma \varphi(\alpha(x+\lambda t))\right] \\
& =0 \\
& \Rightarrow \varepsilon \alpha^{2}\left(\varphi^{\prime \prime}(\alpha(x+\lambda t))+\varphi(\alpha(x+\lambda t))\right) * 0 \\
& \Rightarrow \varphi^{\prime \prime}(\alpha(x+\lambda t))+\varphi(\alpha(x+\lambda t))=0 \\
& \text { or } \varphi^{\prime \prime}(x)+\varphi(x)=0 \\
& \Rightarrow \varphi(x)=A \cos x+B \sin x ; A, B \in \mathbb{R} \\
& \text { And } u(0, x)=\varphi(\alpha x) .
\end{aligned}
$$

\section{Conclusion}

The SBA numerical method permitted us to resolve a few linear partial differential equations modelling diffusion, convection, reaction problems Cauchy type. The SBA method pemitted us to resolve the problems proposed in this paper. It is then a very powerful numerical tool of analysis for the resolution of these Kinds of problems.

\section{References}

[1] K. Abbaoui and Yves Cherruault. The decomposition method applied to the cauchy problem. Kybernetes, 28(1):68-74, 1999.

[2] Bonazebi.J.Yendoula Pare Youssouf Bissanga.G Bassono.F and Some. B. Application of the adomian decomposition method (adm) and the some blaise abbo(sba) method to solving the diffusion-reaction equations. Advances in Theoritical and Applied Mathematics, 9(2):97-104, 2014.

[3] Bakari Abbo N. Ngarasta B.Mampassi B.Some and Longin Some. A new approach of the adomian algoritm for solving nonlinear ordinary or partial differential equations. Far East.J. Math., 23(3):299-312, 2006.

[4] Pare Youssouf Yaro Rasmane Elysée Gouba and Some Blaise. Solving a system of nonlinear equations second kind of volterra by the sba method. Far East.J.Appl.Math, 71(1):43-84, 2012. 
[5] K.Abbaoui and Yves Cherruault. Convergence of the adomian method applied to the nonlinear equations. Math. Comput. Modelling, 20(9):60-73, 1994.

[6] K. Abbaoui N.Ngarasta, B.Some and Yves Cherruault. New numerical study of adomian method applied to a diffusion model. Kybernetes, 31(1):61-75, 2002.

[7] Wazwaz.A.M. Partial differential equations and applications. Netherland Balkema Publisher, 2002.

[8] M.Bagayogo Y.Pare and Y. Minoungou. An approached solution of wave equation cubic damping by homotopy perturbation method (hpm), regular perturbation method(rpm) and adomian decomposition method (adm). J.Math.Res., 31(2):166-181, 2018. 